\title{
Combined bronchoscopy, mediastinoscopy, and thoracotomy for lung cancer: Who benefits?
}

Kwhanmien Kim, $\mathrm{MD}^{\mathrm{a}}$

Thomas W. Rice, $M D^{\mathrm{a}}$

Sudish C. Murthy, MD, $\mathrm{PhD}^{\mathrm{a}}$

Malcolm M. DeCamp, MD

Christopher D. Pierce, $\mathrm{PhD}^{\mathrm{a}}$

Daniel P. Karchmer, MBA $^{\text {a }}$

Lisa A. Rybicki, $\mathrm{MS}^{\mathrm{b}}$

Eugene H. Blackstone, MD ${ }^{a, b}$

An appendix of three tables is available online.
From the Departments of Thoracic and Cardiovascular Surgery ${ }^{\mathrm{a}}$ and Biostatistics and Epidemiology, ${ }^{\mathrm{b}}$ The Cleveland Clinic Foundation, Cleveland, Ohio.

Read at the Eighty-third Annual Meeting of The American Association for Thoracic Surgery, Boston, Mass, May 4-7, 2003.

Received for publication April 30, 2003; revisions requested Oct 6, 2003; accepted for publication Nov 3, 2003.

Address for reprints: Thomas W. Rice, MD, The Cleveland Clinic Foundation, $9500 \mathrm{Eu}-$ clid Avenue/Desk F25, Cleveland, OH 44195 (E-mail: ricet@ccf.org).

J Thorac Cardiovasc Surg 2004;127:850-6

$0022-5223 / \$ 30.00$

Copyright (9) 2004 by The American Association for Thoracic Surgery

doi:10.1016/j.jtcvs.2003.11.020
Objectives: Surgical staging and resection of lung cancer may be done as 1 operation (combined) or 2 (staged). This study evaluates the safety and efficiency of these treatment strategies.

Methods: From 1998 to July 2001, 343 patients underwent bronchoscopy, mediastinoscopy, and thoracotomy without induction chemoradiotherapy by 3 surgeons. Fifty-seven patients were staged and 286 combined. Staged patients had higher clinical stage $(P<.001)$. Propensity-matched groups were compared to adjust for this and other differences. Factors associated with safety and efficiency were identified by propensity-adjusted multivariable analysis.

Results: Mortality and morbidity were similar for both strategies. Efficiency, measured by shorter operative time (1.2 hours) and lower cost $(25 \%)$, was better for combined strategy $(P<.001)$. Hospital stay was similar, but revenue was $12 \%$ higher for the staged strategy $(P<.001)$. In propensity-matched comparisons excluding surgeon, results were similar to the above. Comparisons including surgeon demonstrated similar cost and revenue for both strategies. Increased mortality and morbidity were associated only with patient and tumor characteristics: male gender, worsening Eastern Cooperative Oncology Group performance status, and increasing pathological node classification. All measures of efficiency worsened with increasing pathological classifications. Staged strategy was associated with increased operative time and revenue, while one surgeon and patient smoking history were associated with increased hospital stay and costs.

Conclusions: The combined strategy provides efficient, safe health care for clinically operable lung cancer patients, but it may not be as financially rewarding as the staged strategy. Treatment strategy is only 1 of many determinants of efficiency.

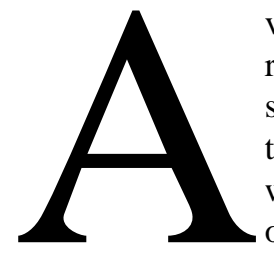

vailability of accurate intraoperative frozen-section diagnosis of regional lymph node metastases led to a strategy of combined surgical staging (bronchoscopy and mediastinoscopy) and resection (thoracotomy) of lung cancer (combined). ${ }^{1}$ This contrasts with a strategy of separating surgical staging from resection as 2 operations (staged). Heterogeneity of practice and a computerized cost-accounting system afforded the opportunity to evaluate the safety and efficiency of these 2 strategies. 


\section{Patients and Methods \\ Patients}

From January 1998 to July 2001, 343 patients underwent bronchoscopy, mediastinoscopy, and thoracotomy for resection of lung cancer. Patients were identified in the Thoracic Surgery Registry, which is approved for research by the institutional review board.

Ninety-eight patients had bronchoscopy and mediastinoscopy followed by thoracotomy as a second procedure (staged); of these, 41 received induction chemoradiotherapy prior to thoracotomy. Three hundred nineteen patients were to have all procedures under the same anesthetic (combined); of these, 297 received it, 286 as sole therapy and 11 after induction chemoradiotherapy. Twentytwo patients $(6.9 \%)$ in the planned combined group had unsuspected mediastinal node metastases at mediastinoscopy and received induction therapy.

A disproportionate number of patients received induction chemoradiotherapy in the staged group (41 of $98,42 \%$ ) versus the combined group (33 of $319,10 \%$ ), which could bias comparisons. Because induction chemoradiotherapy is more prolonged, complicated, and expensive than resection alone,,$^{2-4}$ the analyses concentrated on the 57 staged and 286 combined patients in whom resection was the sole therapy. Patient, tumor, and operative characteristics are listed in Appendix 1. Rationale for resection of pathological stage III and IV lung cancers is presented in Appendix 2 .

\section{Bronchoscopy, Mediastinoscopy, and Thoracotomy}

Staged. Bronchoscopy and mediastinoscopy were outpatient procedures in 54 patients $(95 \%)$. Mediastinal nodes were histologically evaluated from paraffin-embedded specimens stained with hematoxylin and eosin. Patients had thoracotomy after a median of 13 days. Epidural anesthesia was used in 52 patients $(91 \%)$, with the catheter placed prior to induction.

Combined. Two hundred eighty patients (98\%) were admitted the day of surgery; $6(2 \%)$ were in-hospital. Bronchoscopy and mediastinoscopy were performed, and mediastinal nodes were analyzed by frozen section with hematoxylin and eosin staining. Patients with no mediastinal node metastases underwent thoracotomy. Epidural anesthesia was used in 263 patients (92\%).

Postoperative care was identical for both groups.

\section{Safety}

Mortality. Operative mortality was defined as death in-hospital or within 30 days of operation.

Morbidity. Complications were classified as (1) pulmonary, which included reintubation, pneumothorax, adult respiratory distress syndrome, atelectasis requiring bronchoscopy, pneumonia, bronchopleural fistula, pulmonary embolism, and pleural effusion; (2) cardiac, which included arrhythmia and myocardial infarction; or (3) other, which included stroke, vocal cord paralysis, sepsis, and bleeding.

\section{Efficiency}

Operative time. Duration of operation was calculated from anesthesia induction until anesthesia ended. For staged, operative time was the sum of both procedures.

Hospital stay. Total hospital stay was defined as time from admission to discharge date.
Cost. Direct technical costs were costs directly associated with patient care, obtained from the hospital's cost accounting system. Costs included anesthesia, surgery, pathology, nursing (operating room, intensive care unit, hospital, and rehabilitation), pharmacy, radiology, and miscellaneous. Indirect costs and physician fees were not included. Cost of staged was expressed as a percentage of combined. For propensity-matched pairs (see following text), relative cost was the ratio for each matched pair. For overall data, all combinations of patient pairs $(\mathrm{n}=16,302)$ were used and resulting ratios summarized.

\section{Revenue}

Net revenue was payment expected, calculated as charges less contractual insurance adjustments plus patient copay. Revenue of staged was expressed as a percentage of combined.

\section{Comparisons of Strategies}

Unadjusted comparison. Patient characteristics, safety, and efficiency were compared using the chi-square test for categorical variables and appropriate $t$ test, Wilcoxon rank-sum test, or Cox proportional hazards test for continuous variables. All statistical tests were 2-sided.

Propensity-based comparisons. Selection bias was addressed by constructing propensity scores. ${ }^{5,6}$ The probability of undergoing combined versus staged strategy (propensity score) was estimated by logistic regression analysis incorporating 17 variables: gender, age, prior cardiac surgery, prior cancer surgery, cardiac comorbidity, diabetes, weight loss over prior 3 months, Eastern Cooperative Oncology Group (ECOG) performance status, American Society of Anesthesia classification, clinical tumor (T), regional node $(\mathrm{N})$, and distant metastases $(\mathrm{M})$ classifications, use of positron-emission tomography (PET) scan, tissue diagnosis of cancer, hemoglobin, creatinine, and percent predicted forced vital capacity (FVC). Sporadic missing values were imputed by taking the most frequent response category or averaging nonmissing values for continuous variables ( 1 imputed value for diabetes, 3 for tissue diagnosis, 4 for FVC). For each of the 57 staged patients, a well-matched combined patient was sought by propensity score matching. ${ }^{7}$

By comparison without surgeon, the above procedure yielded 54 well-matched pairs (Appendix 3). Surgeon was added to the 17-variable propensity model to yield a second propensity score. Using this score, 44 well-matched pairs were identified (see Appendix 3).

\section{Risk Factors Related to Safety and Efficiency}

Safety. Multivariable logistic regression analysis was used to identify risk factors for morbidity (including mortality) in all 343 patients. Variables considered included 18 used to develop the propensity score plus treatment strategy, type of operation, mediastinal node dissection, tumor histology, number of satellite lung lesions, pathological TNM classifications, pathological stage grouping, and propensity score (surgeon included). Analyses included treatment strategy and propensity score, irrespective of $P$ value. Results are summarized as parameter estimate and standard error with corresponding odds ratio, $68 \%$ confidence limits, and $P$ value. 
TABLE 1. Comparison of early outcome in unadjusted and propensity-matched groups, with and without surgeon

\begin{tabular}{|c|c|c|c|c|c|c|c|c|c|c|c|c|c|c|c|}
\hline \multirow[b]{3}{*}{ Outcome } & \multicolumn{5}{|c|}{ Unadjusted } & \multicolumn{5}{|c|}{ Adjusted, without surgeon } & \multicolumn{5}{|c|}{ Adjusted, with surgeon } \\
\hline & \multicolumn{2}{|c|}{$\begin{array}{c}\text { Staged } \\
(n=57)\end{array}$} & \multicolumn{2}{|c|}{$\begin{array}{l}\text { Combined } \\
(\mathrm{n}=286)\end{array}$} & \multirow[t]{2}{*}{$\boldsymbol{P}$} & \multicolumn{2}{|c|}{$\begin{array}{l}\text { Staged } \\
(n=54)\end{array}$} & \multicolumn{2}{|c|}{$\begin{array}{c}\text { Combined } \\
(n=54)\end{array}$} & \multirow[t]{2}{*}{$\boldsymbol{P}$} & \multicolumn{2}{|c|}{$\begin{array}{c}\text { Staged } \\
(n=44)\end{array}$} & \multicolumn{2}{|c|}{$\begin{array}{l}\text { Combined } \\
(\mathrm{n}=44)\end{array}$} & \multirow[t]{2}{*}{$\boldsymbol{P}$} \\
\hline & No. & $\%$ & No. & $\%$ & & No. & $\%$ & No. & $\%$ & & No. & $\%$ & No. & $\%$ & \\
\hline Mortality & 1 & 1.8 & 6 & 2.1 & .9 & 0 & 0 & 3 & 5.6 & .08 & 0 & 0 & 0 & 0 & - \\
\hline $\begin{array}{c}\text { CL } \\
\text { Morbidity }\end{array}$ & \multicolumn{2}{|c|}{$0.2-5.9$} & \multicolumn{2}{|c|}{$1.2-3.4$} & & \multicolumn{2}{|c|}{$0-3.5$} & \multicolumn{2}{|c|}{$2.4-11$} & & \multicolumn{2}{|c|}{$0-4.3$} & \multicolumn{2}{|c|}{$0-4.3$} & \\
\hline All & 16 & 28 & 85 & 30 & .8 & 14 & 26 & 18 & 33 & .4 & 9 & 20 & 15 & 34 & .15 \\
\hline Pulmonary* & 4 & 7.0 & 26 & 9.1 & .6 & 3 & 5.6 & 8 & 15 & .11 & 2 & 4.6 & 6 & 14 & .14 \\
\hline Cardiac* & $11 \dagger$ & 19 & $51 \dagger$ & 18 & .8 & 10 & 18 & 9 & 17 & .8 & 7 & 16 & 7 & 16 & 1.0 \\
\hline Other* & 5 & 8.8 & 20 & 7.0 & .6 & 4 & 7.4 & 5 & 9.3 & .7 & 1 & 2.3 & 4 & 9.1 & .17 \\
\hline
\end{tabular}

$C L, 68 \%$ confidence limits.

*Not mutually exclusive.

tSixty of the 62 experienced postoperative atrial arrhythmias; 3 experienced a myocardial infarction, including 1 of the 60 .

Efficiency. Because distributions of measures of efficiency were positively skewed, Cox proportional hazards modeling was used to identify risk factors. For hospital stay, data of patients dying in-hospital were included up to time of death, then censored.

\section{Results}

\section{Comparison of Treatment Strategies}

Safety. Operative mortality and morbidity were similar for staged and combined strategies (Table 1).

Efficiency. Median operative time was less for the combined strategy (Figure 1); median hospital stay was similar $(P \geq .1)$. Median relative direct technical costs were greater for staged in the unadjusted and propensity-based comparison without surgeon (Figure 2, A). However, in the comparison with surgeon, costs were similar because combined cost was $15 \%$ higher than in the non-surgeon-matched group, and staged cost was $6 \%$ lower. Median net revenue was higher for the staged strategy (Figure 2, B).

\section{Risk Factors Related to Safety and Efficiency}

Safety. Male gender, worsening ECOG performance status, and increasing pathological $\mathrm{N}$ classification were associated with increased mortality and morbidity (Table 2), but treatment strategy, adjusted for these variables and propensity score, was not $(P=.17)$.

Efficiency. The staged strategy was associated with longer operative time and increased revenue (propensity score was associated with operative time, but is a surrogate for 1 surgeon whose operative time was lower and who had a preference for the combined strategy) (Table 3). Strategy was not associated with hospital stay or cost; however, another surgeon with a preference for the staged strategy was associated with longer hospital stay and higher cost.

\section{Discussion}

\section{Safety}

Accurate staging of lung cancer determines treatment. Combining surgical staging and resection of lung cancer was proposed soon after introduction of mediastinoscopy. However, it has not gained universal acceptance because of concerns about safety and efficiency. This study demonstrates comparable operative mortality and morbidity with either a staged or combined strategy.

These results are typical of modern surgical outcomes, ${ }^{10,11}$ with a $2 \%$ operative mortality and $29 \%$ prevalence of complications, with cardiac complications twice as common as others. Patient and tumor characteristics, not treatment strategy, were the only risk factors for adverse early outcomes.

\section{Efficiency}

Efficiency is performing a task with minimum waste, expense, and unnecessary effort; an efficient treatment is one with high output-to-input ratio. Thus, efficiency was characterized by measurable quantities: operative time, hospital stay, direct technical costs, and net revenue. The combined strategy reduced operative time. Hospital stay was not influenced by strategy but by patient and tumor characteristics and surgeon. A surgeon's postoperative protocols and discharge criteria determine hospital stay and have an impact equal to that of other risk factors for longer stay.

Expenditure of effort is difficult to quantify; we chose the surrogate direct cost. For the practice as a whole and for equivalent patients, costs were less when the combined strategy was used. However, propensity-matched comparisons and multivariable analyses demonstrated that a surgeon's practice could negate cost savings of this strategy. Cost inefficiencies include unnecessary use of expensive equipment and supplies, inefficient use of operative time, lack of discharge planning, and expensive idiosyncrasies. It is also more costly to care for smokers or patients with advanced-stage lung cancers.

Preoperative patient instruction and preparation can reduce cost. Reimbursement is not directly coupled with costs, as demonstrated in this study. Payor algorithms vary. 


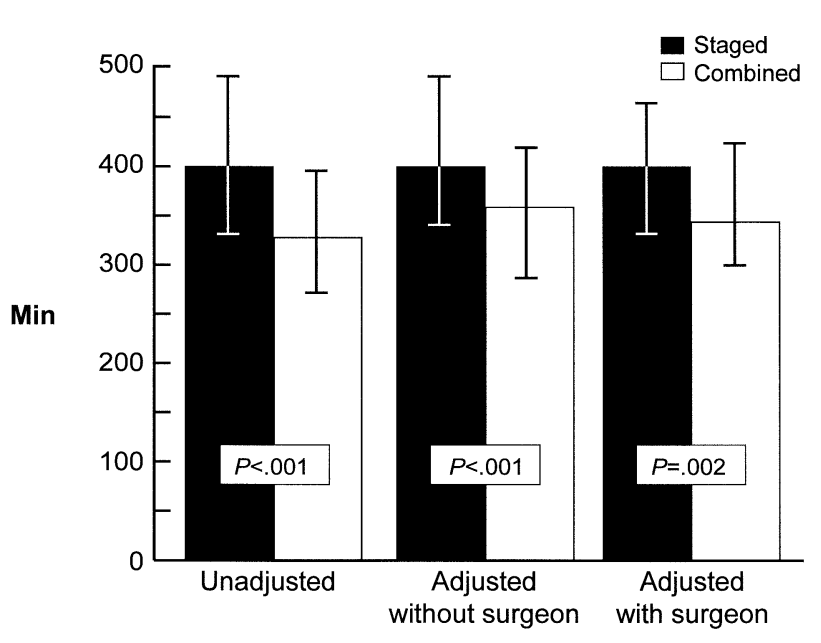

Figure 1. Comparison of cumulative operating time in unadjusted and propensity-matched groups, with and without surgeon. Bar represents median and crossbars represent 15th and 85th percentiles. Min, Minutes.

A staged strategy will be reimbursed as 2 procedures by some payors, but as a single DRG by others. Therefore, payor mix will dictate levels of reimbursement for each strategy. Although reimbursement is increased for sicker patients, in this study reimbursement algorithms did not recognize efficient health care delivery.

\section{Benefits and Disadvantages}

From an institutional perspective, there is potential loss of operating room time with the combined strategy in about $7 \%$ of clinically operable patients with lung cancer who have a positive mediastinoscopy and do not proceed to planned thoracotomy. This "unused" operating time may be absorbed by unexpected needs such as unrealistically scheduled elective surgery, same-day elective surgery, and emergency operations. Dynamic optimization of this fixed resource (operating room time) requires adding this "7\%" factor to the utilization equation. ${ }^{12-14}$

Despite similar mortality and morbidity, the staged strategy exposes all patients to risks of 2 operations, and the combined strategy exposes $7 \%$ of patients to an aborted planned thoracotomy. A psychological benefit may be provided by prompt, same-day treatment decision offered by the combined strategy. The patient care team treats the patient only once; a surgeon can consolidate decision making to 1 episode and can be assured that histologic information is $99.4 \%$ accurate $^{1}$ and mediastinoscopy $94 \%$ negatively predictive; and optimal health care delivery is facilitated by eliminating redundant services. Unfortunately, reduction of costs may not be linked directly to increased revenue.
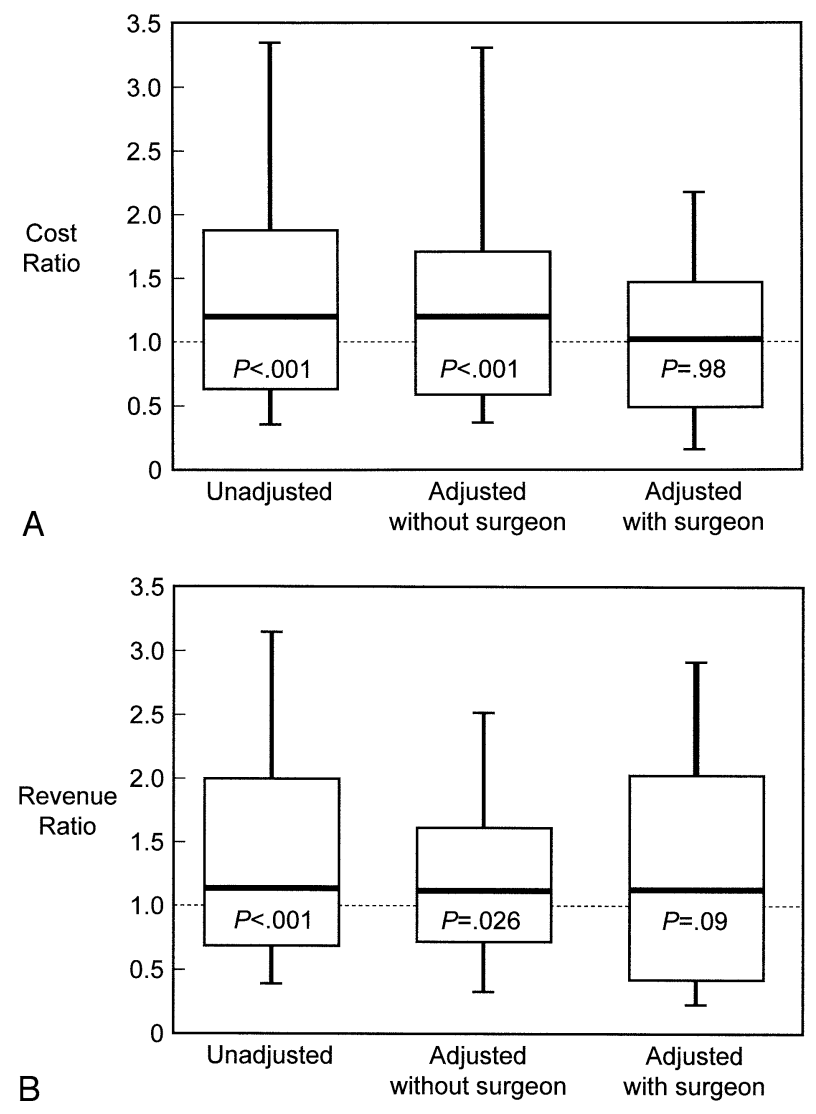

Figure 2. Box and whiskers comparison of monetary measures of efficiency in unadjusted and propensity-matched groups, with and without surgeon, expressed as the ratio of staged to combined strategies. Dashed horizontal line at 1.0 represents cost and revenue of combined approach. Each box encompasses $70 \%$ of the ratios; horizontal line within box represents median ratio; whiskers extend below to 5th percentile and above to 95th percentile of ratios. A, Direct technical cost. B, Revenue.

A disadvantage of the combined strategy is that if it is abandoned, epidural and Foley catheters are needlessly placed, with their attendant risks and costs.

\section{Limitations}

This is a single-institution study and may not be generalizable. It is limited by lack of randomization, which is possible despite inability to blind the team to strategy. Contemporary methods for generating quasi-randomized comparison, as done in this study, are also limited by inability to account for unrecorded variables and to separate confounding between surgeon and strategy. ${ }^{5,6}$ For this reason, we have emphasized that it is the combination of surgical team and strategy that is evaluated in the propensity-matched comparisons, without accounting for surgeon; in the comparisons with surgeon incorporated in the propensity score, both matched comparison and propensity- 
TABLE 2. Incremental risk factors for adverse early outcomes

\begin{tabular}{|c|c|c|c|c|}
\hline Risk factor & Estimate \pm SE & $\mathbf{O R}$ & CL & $\boldsymbol{P}$ \\
\hline Combined strategy & $0.57 \pm 0.41$ & 1.76 & $1.17-2.8$ & .17 \\
\hline Propensity score ${ }^{*}$ & $-0.084 \pm 0.067$ & 0.92 & $0.86-0.98$ & .2 \\
\hline Male & $0.72 \pm 0.26$ & 2.0 & $1.58-2.7$ & .006 \\
\hline Worse ECOG performance status & $0.42 \pm 0.170$ & 1.52 & $1.29-1.80$ & .01 \\
\hline Higher pN† & $0.55 \pm 0.18$ & 1.73 & $1.44-2.1$ & .003 \\
\hline $\begin{array}{l}\text { Intercept } \\
\text { c-statistic }\end{array}$ & $\begin{array}{c}-1.63 \pm 0.55 \\
.67\end{array}$ & & & \\
\hline
\end{tabular}

$C L, 68 \%$ confidence limits; ECOG, Eastern Cooperative Oncology Group; OR, odds ratio; $S E$, standard error.

* Per $10 \%$ increase in score.

tPer 1-level increase, pN0 to pN2.

TABLE 3. Incremental risk factors for decreased efficiency

\begin{tabular}{|c|c|c|c|c|}
\hline Risk factor & Estimate \pm SE & HR & CL & $P$ \\
\hline \multicolumn{5}{|l|}{ Temporal measures } \\
\hline \multicolumn{5}{|l|}{ Longer operative time ${ }^{*}$} \\
\hline Staged strategy & $-0.77 \pm 0.179$ & 0.46 & $0.39-0.55$ & $<.001$ \\
\hline Propensity score & $0.094 \pm 0.034$ & 1.10 & $1.06-1.14$ & .006 \\
\hline Higher pT‡ & $-0.59 \pm 0.161$ & 0.56 & $0.47-0.65$ & $<.001$ \\
\hline Higher pN§ & $-0.42 \pm 0.129$ & 0.66 & $0.58-0.75$ & .001 \\
\hline \multicolumn{5}{|l|}{ Longer hospital stay* } \\
\hline Combined strategy & $-0.199 \pm 0.184$ & 0.82 & $0.68-0.98$ & .3 \\
\hline Propensity score† & $0.0138 \pm 0.033$ & 1.01 & $0.98-1.05$ & .7 \\
\hline Older age $\|$ & $-0.127 \pm 0.058$ & 0.88 & $0.83-0.93$ & .03 \\
\hline Smoker & $-0.49 \pm 0.22$ & 0.61 & $0.49-0.76$ & .03 \\
\hline Higher pNף & $-0.22 \pm 0.095$ & 0.80 & $0.73-0.88$ & .02 \\
\hline Surgeon B & $-0.37 \pm 0.142$ & 0.69 & $0.60-0.79$ & .008 \\
\hline \multicolumn{5}{|c|}{ Monetary measures } \\
\hline \multicolumn{5}{|c|}{ Higher total direct costs* } \\
\hline Staged strategy & $-0.021 \pm 0.190$ & 0.98 & $0.68-1.42$ & .9 \\
\hline Propensity score & $0.0088 \pm 0.036$ & 1.01 & $0.97-1.04$ & .8 \\
\hline Smoker & $-0.63 \pm 0.22$ & 0.53 & $0.42-0.66$ & .004 \\
\hline Higher pT‡ & $-0.41 \pm 0.163$ & 0.67 & $0.57-0.78$ & .01 \\
\hline Higher pN§ & $-0.32 \pm 0.134$ & 0.72 & $0.63-0.83$ & .02 \\
\hline Surgeon B & $-0.30 \pm 0.138$ & 0.74 & $0.65-0.85$ & .03 \\
\hline \multicolumn{5}{|l|}{ Lower net revenue* } \\
\hline Combined strategy & $0.42 \pm 0.183$ & 1.52 & $1.27-1.83$ & .02 \\
\hline Propensity score† & $-0.0075 \pm 0.032$ & 0.99 & $0.96-1.02$ & .8 \\
\hline Lower creatinine\# & $-0.54 \pm 0.21$ & 0.58 & $0.48-0.71$ & .008 \\
\hline Lower pT‡ & $-0.50 \pm 0.164$ & 0.61 & $0.52-0.72$ & .002 \\
\hline
\end{tabular}

$C L, 68 \%$ confidence limits; $H R$, hazard ratio; $S D$, standard deviation; $S E$, standard error.

*Negative estimates and hazard ratios less than 1.0 correspond to less desirable outcome.

tPer $10 \%$ increase in score.

‡рT3-4 vs pT1-2.

§pN1-2 vs pNO.

|Per 10-year increase.

ПPer 1-level increase, pN0 to pN3.

\#Per $1 \mathrm{mg} \cdot \mathrm{dL}^{-1}$ increase.

adjusted multivariable analysis highlight the importance of the surgeon in the cost-benefit balance.

\section{Requirements and Indications for Combined Strategy}

The combined strategy requires accurate clinical staging. Using history, physical examination, routine blood work, and computed tomography, but with minimal use of PET, only $7 \%$ of patients had unsuspected $\mathrm{pN} 2$ or $\mathrm{pN} 3$ disease at mediastinoscopy. This strategy also requires rigorous mediastinoscopy and accurate and efficient histologic evaluation. In this study group, mediastinoscopy was $95 \%$ accurate, $75 \%$ sensitive, $100 \%$ specific, $100 \%$ positively predictive, and $94 \%$ negatively predictive (Appendix 2). No frozen-section diagnosis was reversed by 
routine histologic evaluation. In our previous experience, false-negative frozen section analysis occurred in $0.6 \%$ of patients. ${ }^{1}$

Assuming these requirements for a combined strategy are in place, any patient who is medically operable and has clinical stage I or II non-small cell lung cancer should be offered a combined strategy. If mediastinoscopy demonstrates unanticipated mediastinal nodal metastases, this strategy should be abandoned. Because there is an obligatory waiting period during frozen-section analysis of sampled mediastinal nodes, the potential time saving of the combined strategy can be lost if this period is not used preparing for thoracotomy or if frozen-section analysis is inefficient.

\section{References}

1. Gephardt GN, Rice TW. Utility of frozen-section evaluation of lymph nodes in the staging of bronchogenic carcinoma at mediastinoscopy and thoracotomy. J Thorac Cardiovasc Surg. 1990;100:853-9.

2. Stamatis G, Djuric D, Eberhardt W, Pottken C, Zaboura G, Fechner S, et al. Postoperative morbidity and mortality after induction chemoradiotherapy for locally advanced lung cancer: an analysis of 350 operated patients. Eur J Cardiothorac Surg. 2002;22:292-7.

3. Roberts JR, Eustis C, Devore R, Carbone D, Choy H, Johnson D. Induction chemotherapy increases perioperative complications in patients undergoing resection for non-small cell lung cancer. Ann Thorac Surg. 2001;72:885-8.

4. Deslauriers J. Medical complications of combined surgical and nonsurgical therapy. Curr Opin Oncol. 2000;12:127-31.

5. Rosenbaum PR, Rubin DB. The central role of the propensity score in observational studies for causal effects. Biometrika. 1983;70:41-55.

6. Blackstone EH. Comparing apples and oranges. J Thorac Cardiovasc Surg. 2002;123:8-15.

7. Parsons L. Reducing bias in a propensity score matched-pair sample using greedy techniques. Cary (NC): SAS Institute, Inc; 2001.

8. Kirschner PA. A coordinated surgical approach to diagnosis and treatment of lung cancer. Chest. 1970;57:214-5.

9. Falor WH. One-stage combined diagnosis and therapy of intrathoracic tumors. Am Rev Respir Dis. 1967;95:59-65.

10. Ginsberg RJ, Hill LD, Eagan RT, Thomas P, Mountain CF, Deslauriers J, et al. Modern thirty-day operative mortality for surgical resections in lung cancer. J Thorac Cardiovasc Surg. 1983;86:654-8.

11. Shields T, Ponn RB. Complications of pulmonary resection. In: Shields T, LoCicero J III, editors. General thoracic surgery. 5th ed. Philadelphia: Lippincott Williams \& Wilkins; 2000. p. 481-505.

12. Bryson AE Jr. Dynamic optimization. Boston: Addison-Wesley; 1999

13. Chiang AC. Elements of dynamic optimization. Prospect Heights (IL): Waveland Press; 1999.

14. Kamien MI, Schwartz NL. Dynamic optimization. St Louis: Elsevier; 2000.

\section{Discussion}

Dr Bryan F. Meyers (St Louis, Mo). Congratulations on your work. I learn something from every paper that comes out of the Cleveland Clinic, and this one is no exception.

This isn't really a question. It's just a dart to harass you a little bit and put you off guard. I would say that the safety issue on this paper is sort of a null issue. I would conclude that it's very safe to have lung surgery at the Cleveland Clinic. You had only 7 deaths among 343 patients, and you would probably need several thousand patients to show a clinically meaningful difference in mortality between 2 strategies, regardless of what those strategies were. Furthermore, there are no logical or plausible events that would affect the safety of the operation and that would be caused by breaking the bronchoscopy and the mediastinoscopy away from the thoracotomy. It's intuitively not plausible, and you don't have the power to assess safety. So why not just call this an efficiency paper, period?

Dr DeCamp. Dr Meyers, I appreciate that comment. I'm officially off balance. I think in the early literature, when mediastinoscopy was introduced as a way of staging operable lung cancer, there was an issue of safety of performing staging and resection all as 1 procedure when the accuracy of frozen-section analysis was in question. It clearly takes a longer operative time to do a combined procedure. If you have an institution that can turn these frozen sections around quickly, then the patient is asleep just a short time longer and has less fluid infused. Early on, however, it was our initial impression that some of the combined patients got a lot more intraoperative fluid and perhaps had more pulmonary complications. So we initially thought about looking at this, and we had a hypothesis that perhaps there was a safety issue. We were happy to see that there wasn't.

It's true that both anatomic lung resection and mediastinoscopy are pretty safe in our hands and that the primary thrust of this work is the economic analysis; nevertheless, the safety issues are important. The message to the surgeon is, as long as you can do them both safely, you can do them at the same time or staged, whatever you want.

Dr Meyers. You have chosen to limit your analysis to patients who have had all 3 procedures: bronchoscopy, mediastinoscopy, and thoracotomy. In the paper, $7 \%$ of the patients who were intended to have bronchoscopy, mediastinoscopy, and thoracotomy had a positive mediastinoscopy and then were excluded from the analysis. Because there was a tendency toward larger tumors in the combined group, those patients probably had a higher rate of positive mediastinoscopy. It seems like you have biased the analysis, because the bronchoscopy/mediastinoscopy, followed a week later by thoracotomy, is a strategy that works out better in the case of a positive mediastinoscopy: you are more efficient with operating time. In the combined plan, if you have a positive mediastinoscopy, you're left with a hole in your operating schedule. So I think you've biased the efficiency analysis a bit by including only patients who had all 3 procedures and excluding the patients who a stand-alone bronchoscopy.

Dr DeCamp. I would disagree with the suggestion that we biased the analysis. Propensity matching is designed, in fact, to correct selection bias. It is true that larger tumors are more likely to have a positive mediastinoscopy. Those patients scheduled for combined approach were clinically felt not to have N2 disease, but $7 \%$ of the time there were unexpected $\mathrm{N} 2$ nodes. Those patients were excluded because when we get into the cost/revenue issue, we wanted to avoid the morass of induction therapy and the increasing costs related to that. So I think in terms of the actual analysis, we've tried to make it as clean as we can. In the staged bronchoscopy, mediastinoscopy, and thoracotomy group, the positive mediastinoscopies are also excluded from the cost/revenue analysis because, again, we excluded patients who had advancedstage disease and who went off and had chemotherapy. So I think the comparison is valid.

Your point is well taken that an unsuspected positive mediastinoscopy is an annoyance sometimes to the surgeon because you've blocked 3 to 4 hours of time and what are you going to fill that operating room time with? We try to make the point in the 
article that this may not be a good strategy for all institutions, but if you have an institution that has some flexibility and a reasonable volume of unscheduled things that need to get done, you might be able to make up that $7 \%$ time. If you're in solo practice and your next operative day is a week away, then this may not be a particularly effective strategy.

Dr Meyers. You talked about the neatness of the analysis, and it is neat and it's very carefully and accurately done, but I just wonder if all the propensity analysis and greedy matching and balancing can overcome the skepticism that might be in the room. If you just took the 44 patients per group that ended up in the propensity-matched analysis and randomized them into 2 groups, might you not have been able to have an actual clinical trial that everybody could sink their teeth into?

Dr DeCamp. One of the conclusions that we draw from this experience is that you could certainly do a prospective randomized trial and try to tease apart what we're trying to do retrospectively with propensity matching. That's one of the values of this kind of statistical analysis. What we tried to show was that in my hands a staged approach was cost effective and in my partner's hands a combined approach was cost effective as long as we applied our own clinical judgment to the particular patient. I think we would all agree that big tumors or big nodes are more likely to have positive mediastinoscopies and are better approached in a staged fashion.

Dr Michael T. Jaklitsch (Boston, Mass). I thought it was a great presentation, and this analysis makes me comfortable with your decision to do a combined approach. I do a staged approach. But this analysis doesn't convince me to abandon my way and adopt a combined approach, because, again, it's a point that $\mathrm{Dr}$ Meyers already brought up: in this world of limited resources, my personal most limited resource is operating room time. If I have to block out thoracotomy time for every patient who is going for a mediastinoscopy and it's a negative mediastinoscopy, I'm going to start pushing out my waiting time to 3 weeks, 4 weeks, and then they are all going to see Dave Sugarbaker because he can get them in quicker. The issue is that it is very predictable how long a mediastinoscopy takes whether it's going to be positive or negative and it is very predictable how long a thoracotomy is going to take.
Combining the two injects doubt into my schedule. I am not convinced that the combined approach suits my practice.

Dr Sugarbaker. Mike, do you have a question or just a comment?

Dr Jaklitsch. A comment.

Dr DeCamp. Thank you, Dr Jaklitsch, for that insightful comment. I want to make the point that this analysis in terms of cost and revenue was done from the perspective of the hospital, not for the surgeon. So your waiting time wasn't given any monetary value, I'm sorry to say, in this analysis.

Dr Mark J. Krasna (Baltimore, Md). I have a question actually relating to what we in Maryland call the BLT, and that is for a left-sided tumor where you have a suspicion of an enlarged aortopulmonary window node on computed tomography. In that clinical scenario we have evolved the following sequence-a bronchoscopy, mediastinoscopy, with Chamberlain/thoracoscopy - at 1 sitting, and because that would be just too long to do together, we would perform your thoracotomy the next week. Some people advocate doing the bronchoscopy/mediastinoscopy at 1 sitting and then bringing the patient back for the thoracoscopy/ thoracotomy. I wonder if you have tried to do the bronchoscopy, mediastinoscopy, left thoracoscopy, and lobectomy at the Cleveland Clinic, and how many of those can you get done in 1 day?

Dr DeCamp. I don't have the data from this analysis to tell you how many of these were left upper lobe tumors where that would be a strategy that we would have to invoke. I can tell you that it's my practice to still do cervical and anterior mediastinoscopy for left upper lobe tumors.

I occasionally approach staging left upper lobe disease with just the video-thoracoscope and look at the aortopulmonary window and make the leap of faith judgment that if there is no N2 disease at levels 5 and 6 , then there is not contralateral N3 disease. I don't think I'm speaking on my partner's behalf in that regard.

Dr Krasna. Can you do that at the same time as the bronchoscopy/mediastinoscopy/staging thoracoscopy?

Dr DeCamp. Yes, though sometimes I just do the thoracoscopy and don't do the mediastinoscopy for peripheral left upper lobe T1 tumors. 